\title{
Interculturalidad: ¿Quo vadis? Desde la aparición de la Interkulturelle Germanistik hasta la normativa europea vigente con especial énfasis en el contexto español
}

\author{
Nieves Rodríguez Pérez / Bárbara Heinsch \\ Universidad de Oviedo \\ nirope@uniovi.es / heinschbarbara@uniovi.es \\ https://dx.doi.org/10.12795/futhark.2013.i08.13
}

\begin{abstract}
This article focuses, first of all, on the revision of the concept of interculturality and its evolution in the field of German Studies over the last decades. This necessarily includes a revision of the most important definitions of culture and its derivatives, pluri- and multi-, within the context of the new socio-cultural constellations in European societies. Secondly, the "Boom" of interculturality will be analyzed, both regarding research and the new European regulations about language policy (Common Framework, White Paper on Intercultural Dialogue etc.) which reshape the profile of the new generations of academics and bring about a change in the paradigm. The next step will be to determine the importance of this phenomenon in the context of Spanish regulations. A deeper analysis of the concept of "intercultural competence" in the examined documents shows that it lacks concrete contents, because the term is often instrumentalized for political and ideological reasons. Its impact upon the scientific-academic field is still weak, in spite of its inclusion in many study programmes. Lastly, the necessity of concrete criteria to provide an education in intercultural competences will be highlighted. These criteria should not only be determined with an eye on employability, but they should also, and first of all, reflect the results of the latest studies concerning interculturality, especially those hinting at a "tertiary culture".

Keywords: Intercultural competence, interculturality, Intercultural German Studies, regulation.

Resumen: El presente artículo aborda, en primer lugar, una revisión del concepto de interculturalidad y su evolución en las últimas décadas en las ramas relacionadas con la Filología Alemana (Interkulturelle Germanistik, Interkulturelle Kommunikation, Deutsch als Fremdsprache), lo cual implica necesariamente una revisión de las principales definiciones de cultura y sus derivados pluri- y multi- en el contexto de las nuevas constelaciones
\end{abstract}


socioculturales en las sociedades europeas. En segundo término, se analiza el "boom" de la interculturalidad tanto en la investigación como en las nuevas normativas europeas en materia de política lingüística (Marco Europeo, Libro Blanco sobre el diálogo intercultural, etc.), que determinan el perfil de la futura generación de académicos e inducen a un cambio de perspectiva, para finalmente definir el lugar que ocupa este fenómeno en la normativa española. Al ahondar en el concepto de competencia intercultural se observa que en los documentos oficiales analizados éste carece de contenidos concretos - precisamente porque a menudo es un concepto instrumentalizado -, y su repercusión en el campo científico-académico no es más que incipiente a pesar de su inclusión en muchos programas de estudio. En último lugar, se destaca la necesidad de criterios concretos para la formación en competencias interculturales que no sólo se determinen bajo la perspectiva de la empleabilidad, sino también, y ante todo, sean reflejo de los resultados de investigación más recientes en el campo de la interculturalidad, especialmente aquéllos que apuntan a una "cultura terciaria".

Palabras clave: Competencia intercultural, interculturalidad, Interkulturelle Germanistik, normativa.

\section{Nota preliminar}

Tanto el concepto de interculturalidad como el de cultura están al orden del día en muchas ramas del conocimiento, en la economía, la política y en contextos sociales. Proliferan los textos que intentan ahondar en su naturaleza o abrir nuevas líneas de investigación - con más o menos éxito - y aquéllos que instrumentalizan estos conceptos para fines ideológicos, políticos y económicos. En un tiempo de fuertes movimientos migratorios, de transformación paulatina del mundo en una aldea global y de pérdida de soberanía de los Estados nacionales, se observan a la vez corrientes que contrarrestan los procesos de homogenización profundizando en lo que diferencia y da identidad: lo regional y local, la propia lengua y cultura, por minoritaria que sea. No cabe duda de que la aparición de realidades y conceptos como Interkulturelle Germanistik, interkulturell e Interkulturalität obedece a una necesidad histórica que se observa claramente tanto en Alemania a partir de los años 70 como en España dos décadas más tarde.

Antes de ofrecer una breve recopilación de los datos históricos que contribuyeron a la constitución tanto de la Interkulturelle Germanistik como del Alemán como Lengua Extranjera revisamos las investigaciones más destacadas acerca del concepto de cultura, puesto que es el término clave para el estudio de la comunicación intercultural. 


\section{Referentes conceptuales: Aproximación al concepto de cultura}

En el mundo interconectado en el que nos desenvolvemos, donde el lenguaje surge consciente o inconscientemente según los hábitos lingüísticos de cada grupo social, en el que convive un conglomerado de estructuras sociales, se hace necesaria una reflexión profunda de cómo generar y fomentar la comunicación intercultural. El ámbito académico, en la actualidad un espacio multicultural, es un lugar privilegiado para hacer conscientes las divergencias y las convergencias culturales que obstaculizan o facilitan la aceptación de otros modos de pensar y entender el mundo.

El interés por la cultura se gesta en la Antigüedad, entendida como el encuentro entre dos grupos culturales. Sin embargo, hasta la llustración francesa no surge el primer intento de dar una explicación científica a este constructo. El término procede de las voces latinas cultus y colere (cultivo, culto) que aludían a la acción humana (la labranza), posteriormente adquiere un sentido metafórico con el que se hacía referencia a una característica de los humanos (personas, colectividades o países «cultivados»). En los siglos XVI-XVIII adquiere un componente dinámico, remitía a la descripción e interpretación de la dinámica de la realidad social en la configuración del Sistema Mundo y se situaba en el campo semántico de cambio, progreso y evolución. Este concepto germina en Alemania con el término Kultur que hace referencia a las diferencias nacionales, universales, emocionales y espirituales frente a lo racional (Kumper 24). En la actualidad, civilización alude a una fase de la evolución social mientras que cultura es una realidad universal.

El término cultura es un concepto muy amplio que abarca diferentes ámbitos, el sociológico, económico, político, tecnológico, científico y espiritual, lo cual genera una gran dificultad al intentar dar una definición que abrace todos los aspectos de la vida. Prueba de ello se evidencia en las investigaciones de Kroeber y Kluckhohn que analizaron 164 definiciones del término según la conceptualización procedente de antropólogos, sociólogos, psiquiatras y otros.

Desde la antropología, que ha asumido este campo de estudio, Edward B. Tylor, autor de la primera definición antropológica de cultura, inspira el punto de partida para la historia oficial del término:

"La cultura o civilización, en sentido etnográfico amplio, es aquel todo complejo que incluye el conocimiento, las creencias, el arte, la moral, el derecho, las costumbres y cualesquiera otros hábitos y capacidades adquiridos por el hombre. La situación de la cultura en las diversas sociedades de la especie humana, en la medida en que puede ser investigada según principios generales, es un objeto apto para el estudio de las leyes del pensamiento y la acción del hombre". (29) 
Kumper (75) encuentra que esta definición es una enumeración de elementos de contenido cultural sin coherencia, además, el autor no distingue entre cultura y organización social.Malinowski, padre de la antropología moderna, retoma los planteamientos de Tylor relativos a la función social y añade:

"Los artefactos, los comportamientos aprendidos, las ideas, las costumbres, los valores que se transmiten socialmente. No se puede comprender realmente la organización social sino como parte de la cultura, todas las direcciones específicas de investigación que tienen como objeto las actividades, los logros, las ideas y las creencias humanas se pueden encontrar y fecundar recíprocamente en un estudio de composición de la cultura." (21)

Podemos determinar que la cultura es parte específica del género humano y que, además, las culturas se encuentran en cada grupo humano y forman parte de las características de cada uno de ellos. Los antropólogos Bates y Plog matizan que la cultura es aprendida y se trasmite de generación en generación, no es un atributo individual sino que es compartida. Sin embargo, en su definición no se contempla el carácter dinámico provocado por el encuentro entre hablantes de otras culturas:"a system of shared beliefs, values, customs, behaviors, and artifacts that the members of society use to cope with their world and with one another, and that are transmitted from generation to generation through learning" (7).

Ruiz Román, en su texto sobre educación intercultural, resume en dos enunciados este enfoque: "a) La cultura es un conjunto de significados adquiridos y construidos. b) El hombre adquiere y construye estos significados como miembro de una (o unas) comunidad(es), y no exclusivamente como miembro de la Comunidad" (14).

Desde el ámbito de la psicología social, Hofstede (10) entiende que cada individuo posee modelos de pensamiento y de actuación que ha ido aprendiendo a lo largo de su vida, especialmente en la etapa de la infancia. Estos modelos los concibe como el software de la mente: cada persona está dotada de diversas capas (lingüística, étnica, generacional, nacional, regional, etc.) de programación mental que condiciona su conducta. En consecuencia, las dimensiones de las diversas culturas nacionales (a través de las migraciones, los medios de información, viajes...) influyen y se incorporan a la otra sociedad provocando una nueva configuración mental. Entendido de esta forma, toda cultura se ha ido formando a partir de los contactos con otros modos de pensar, sentir y actuar.

Teniendo en cuenta las diferentes perspectivas desde las que se ha realizado el estudio del concepto de cultura, podemos definir este término como el conjunto de conductas aprendidas que se transmiten a las siguientes generaciones, con significados que se comparten; es dinámi- 
ca en el sentido de que cambia debido a las influencias de los préstamos. Las interpretaciones que cada individuo realiza de los acontecimientos sociales y naturales desencadenan una determinada conducta. Además, todas las culturas poseen códigos lingüísticos verbales y no verbales, un lenguaje propio, un sistema de símbolos compartidos que permite a los individuos manejarse en el medio. A este respecto, Asunción-Lande manifiesta lo siguiente: "Esto les permite interactuar con otras personas y hacer predicciones de expectativas y acontecimientos. En algunas ocasiones se conoce a este marco de referencia como 'identidad cultural" (6). Casal Iglesias añade que uno de los beneficios de la experiencia del encuentro intercultural es forzarnos a ser conscientes de ello, lo que nos permite percibir y conocer la propia cultura, alguno de cuyos aspectos pueden estar ocultos para nosotros.

Dentro del área de la didáctica no resultó fácil enlazar el aprendizaje de un idioma con los estudios culturales, como manifiestan Barro, Jordan y Roberts:

"La lingüística aplicada y la enseñanza de idiomas generalmente han buscado sus marcos teóricos y conceptuales en la lingüística y la psicología, mientras que la antropología, merecedora de la pretensión de ser la disciplina que más va asociada al estudio de las culturas, se ha encontrado marginada. El resultado ha sido que la "cultura», en vez de debatirse en la bibliografía de la pedagogía de la lengua, ha sido insertada tal cual en los libros de textos de idiomas. Las referencias culturales tienden a manifestarse como afirmaciones esencialistas e irreflexivas, casi totalmente carentes de sentido de agencia individual; por ejemplo, «los franceses sí están más orientados hacia la familia que los ingleses»." (83)

En la literatura especializada en didáctica de lenguas extranjeras, el término "cultura" aparece en diferentes vocablos: multiculturalidad, pluriculturalidad, interculturalidad e incluso existen equiparaciones entre los términos. Aunque en todos ellos aparece el término cultura, que los une y los provee de un sentido común, existen diferencias que requieren su esclarecimiento.

\section{De la multiculturalidad y pluriculturalidad hacia la interculturalidad}

Rodrigo Alsina establece una diferencia clara entre ambos conceptos. Por multiculturalidad entiende la coexistencia de distintas culturas en un mismo espacio real, mediático o virtual, mientras que interculturalidad estaría dirigida a las relaciones que se dan entre las mismas. En palabras del autor:

"El multiculturalismo marcaría el estado, la situación de una sociedad plural desde el punto de vista de comunidades culturales con identidades diferen- 
ciadas. Mientras que la interculturalidad haría referencia a la dinámica que se da entre estas comunidades culturales". (171)

Casanova aborda ambos conceptos en la misma línea. Según él, la multiculturalidad se refiere únicamente a la coexistencia de varias culturas en un espacio determinado, mientras que la interculturalidad

"supone, en principio, que la coexistencia cultural pase a convivencia, con absoluto respeto a las características culturales de cada grupo. Es decir: ni subordinación, ni integracionismo ni asimilacionismo. Sí, por supuesto, respeto y adaptación a las normas sociales que permiten, favorecen y facilitan esa convivencia deseada, pero manteniendo, a ser posible, las peculiaridades importantes de las culturas que, en un momento dado, integran un supragrupo cultural. La convivencia cultural se ve como un enriquecimiento mutuo y no como amenaza a ninguna de las culturas." (12-13)

La definición de Lluch y Salinas es aún más amplia pues describe cinco elementos que caracterizan el concepto de interculturalidad: reconocimiento de la diversidad, defensa de la igualdad, fomento de la interacción, dinámica de la transformación social y promover procesos educativos que plantean la interacción cultural en condiciones de equidad.

El paso de multicultural a intercultural, según Manzini, se desarrolló a través de una renegociación continuada de los roles y espacios, mediante un discernimiento de los valores que orientan los procesos de síntesis.En definitiva, se trata de las percepciones que se tienen del otro, y de las que el otro tiene del contrario. La interculturalidad supone una relación de simetría entre culturas, no de intentar fundir las identidades de las culturas involucradas, sino de aceptar las diferencias, reforzándolas y enriqueciéndolas.

En definitiva, podemos concluir que el término "multiculturalidad" hace referencia a la coexistencia de varias culturas diferentes, sin ahondar en las relaciones entre ellas, mientras que el concepto de "interculturalidad" alude a la interacción, la comunicación, negociación y enriquecimiento entre culturas.

Paralelamente a estos conceptos encontramos "multilingüismo" y "plurilingüismo", el primero, según el Marco común europeo de referencia para las lenguas (Consejo de Europa 2002), hace alusión a la coexistencia de distintas lenguas en una comunidad determinada, en cambio, el plurilingüismo sugiere la integración de las lenguas en el individuo y en extensión, en la sociedad. Al igual que se aprehende una lengua, se integra su "conocimiento cultural" y como resultado se adquiere la competencia plurilingüe y pluricultural, con otras palabras, la competencia plurilingüe hace referencia a la capacidad de participar en una relación intercultural y de emplear la lengua para fines comunicativos. El Marco de referencia define lo siguiente: 
"el enfoque plurilingüe enfatiza el hecho de que conforme se expande la experiencia lingüística de un individuo en los entornos culturales de una lengua, desde el lenguaje familiar hasta el de la sociedad en general y después hasta las lenguas de otros pueblos (ya sean aprendidas en la escuela, o en la universidad o por experiencia directa), el individuo no guarda estas lenguas y culturas en compartimentos mentales estrictamente separados, sino que desarrolla una competencia comunicativa a la que contribuyen todos los conocimientos y experiencias lingüísticas y en la que las lenguas se relacionan entre sí e interactúan". (4)

Dada la creciente importancia de la relación entre culturas y la necesidad de pasar de las reflexiones teóricas a un nuevo campo de acción, que quedó patente en el nacimiento de la Interkulturelle Germanistik como enriquecimiento de la Germanística tradicional alemana, realizamos a continuación una breve revisión de sus orígenes.

\section{Los inicios de la Interkulturelle Germanistik}

La Interkulturelle Germanistik nace en Alemania a raíz de un encargo de la Universidad de Heidelberg dirigido en 1970 a uno de sus profesores, Alois Wierlacher, que consistía en elaborar

„ein Konzept für ein gegenwartsbezogenes Deutsch- und Deutschlandstudium ausländischer, vornehmlich US-amerikanischer undergraduates [...], das ihren Interessen am Erwerb sprach- und deutschlandkundlicher Kenntnisse gerechter werden konnte als die im damaligen Kontext der deutschen Germanistik üblichen und möglichen Fachstudienangebote."(Wierlacher a, 1)

Al mismo tiempo se distinguió por primera vez en la historia de la Germanística entre Grundsprachenphilologie y Fremdsprachenphilologie, es decir, entre Mutter- y Fremdsprachengermanistik (cf. Wierlacher a, 2), lo cual facilitó el reconocimiento y la integración de las necesidades y la alteridad cultural de la Germanística fuera de Alemania, que hasta entonces era considerada comoAuslandsgermanistik, un anexo a la Germanística alemana. Se aspiraba a un "Miteinander kulturdifferenter Perspektiven" (Wierlacher a, 4), para lo que era preciso cambiar de mentalidad científica y, en consecuencia, revisar los métodos de enseñanza del Alemán como lengua extranjera al uso. Esta revisión crítica quedó reflejada en 1977 en el Mannheimer Gutachten. Se produjo un cambio de paradigma visible - entre otras cosas - en la constitución, en 1984, de la Gesellschaft für interkulturelle Germanistik: „Dieser Wechsel leitete, im historiographischen Rückblick gesehen, sowohl den cultural turn der Geisteswissenschaft als auch den xenological turn der Fremdsprachenphilologien ein" (Wierlacher a, 5).

Aparece, pues, el vocablo interkulturell, utilizado por primera vez por Heinz Göhring en 1975 en su contribución al primer número de Jahrbuch 
Deutsch als Fremdsprache; la expresión Deutsch für Ausländer se convierte en Deutsch als Fremdsprache y la pedagogía cambia su „Defizithypothese (den ausländischen Kindern fehlen die sprachlichen und kulturellen Fertigkeiten, um ohne Störung in der deutschsprachigen Schule mithalten zu können)" (Krumm 413) por una "Differenzhypohtese" (id.) que les permite aportar su propia biografía lingüística y cultural en el aula; la competencia intercultural se convierte en un objetivo de la enseñanza del Alemán como lengua extranjera, y se requiere adaptar - no exportar - los métodos. De ahí que en el mercado español se encuentren en la actualidad un número más elevado de manuales de alemán adaptados a hispanohablantes que hace varios lustros.

\section{El "boom" de la interculturaliad}

\subsection{En la investigación}

Lo propio y lo ajeno (dicotomía tematizada ya en 1945/46 por Leo Spitzer) subyace a cualquier reflexión sobre la interculturalidad y ha provocado una creciente avalancha de publicaciones sobre el tema. Para Földes, cultura e interculturalidad son hoy "Schlagworte und Appellbegriffe, [die] über eine prestigeträchtige Semantik [verfügen]" (503), pero que carecen a menudo de definición y fundamento científico:

'Über die Black Box ,Interkulturalität' überwiegen doch eher deklarative Erklärungen statt schlüssiger Theoreme und verifizierbarer Ergebnisse. Dies trifft auch auf den Bereich Deutsch als Fremdsprache/Zweitsprache (DaF/DaZ) zu, der dem Mega- oder gar Gigathema Interkulturalität lediglich an der Oberfläche, mit Blick auf die Praxis der Sprach- und Literaturvermittlung Aufmerksamkeit schenkt. Daher kann man als ein weiteres Forschungsdesiderat feststellen, dass die (DaF-/DaZbezogene) Linguistik - im Gegensatz zu vielen anderen Disziplinen [...] - die Interkulturalität als erkenntnisleitende Perspektive wissenschaftlich bislang relativ selten aufgegriffen hat." (Földes 504)

Alexander Thomas, en su trabajo sobre el aprendizaje intercultural, también destaca - a pesar del „inflationären Gebrauch des Ausdrucks 'interkulturelles Lernen'” (279) - la ignorancia sobre dicho concepto y la falta de consenso entre los investigadores.

Si bien ya existen nuevos planteamientos que trascienden la dicotomía tradicional implícita en la manera occidental de pensar la interculturalidad - y que bien merecen otro estudio -, sólo representan un punto de partida. Para ladidáctica del aprendizaje de las lenguas y su fundamentación científica cobra especial importancia el concepto de competencia intercultural, normalmente condicionado por las intenciones y 
expectativas de entidades educadoras y empleadoras que influyen de manera decisiva en la formulación de los objetivos.

Stefanie Rathje recoge en su estudio de 2006 el estado del debate actual en las investigaciones de habla alemana sobre la competencia intercultural y destaca la gran heterogeneidad en el mismo, debida en la mayoría de los casos a un "teils erbitterte[n] Streit um die Grundlagen interkultureller Kompetenz" (Rathje 3). Uno de los cuatro puntos conflictivos que cita la autora es cuál ha de ser el objetivo de la competencia intercultural:

"Die Stellungnahmen zu Zielvorstellungen interkultureller Kompetenz bewegen sich zwischen eher ökonomisch orientierten Konzepten, die vor allem Effizienzgesichtspunkte in den Vordergrund stellen und eher geisteswissenschaftlichen, bzw. erziehungswissenschaftlichen Ansätzen, die Effizienzerwägungen gegenüber skeptisch eingestellt sind und vor allem den Aspekt menschlicher Weiterentwicklung in der interkulturellen Interaktion betonen."(4)

Puesto que la competencia intercultural consta entre los objetivos de aprendizaje de un gran número de grados universitarios, surgen automáticamente las siguientes preguntas: ¿En qué consiste, cómo se desarrolla y cómo se enseña? ¿Qué respuesta dan los documentos oficiales?

\subsection{En la normativa europea}

El Marco común europeo de referencia para las lenguas (Consejo de Europa 2002), documento fundacional para la enseñanza-aprendizaje de las lenguas, fruto del trabajo desde 1971 de muchos expertos en la enseñanza de las lenguas, provenientes de toda Europa y más allá, y compendio de la filosofía que subyace a la política lingüística de la Unión Europea, formula objetivos muy claros con respecto a la diversidad cultural y lingüística y propone formas de actuación de los europeos ante ella:

“• Propiciar el entendimiento, la tolerancia y el respeto mutuos respecto a las identidades y a la diversidad cultural por medio de una comunicación internacional más eficaz.

- Mantener y desarrollar la riqueza y la diversidad de la vida cultural europea mediante un mejor conocimiento mutuo de las lenguas nacionales y regionales, incluidas las menos estudiadas.

- Satisfacer las necesidades de una Europa multilingüe y multicultural desarrollando considerablemente la habilidad de los europeos para comunicarse entre sí superando las barreras lingüísticas y culturales." (3) 
No obstante, si buscamos entre las Kann-Beschreibungen - descriptores de lo que "Puede hacer" el usuario de la lengua - referencias a la competencia intercultural, no encontramos ni una sola, ni siquiera el término. Es más, en todo el texto aparece sólo dos veces la palabra "competencia intercultural" sin aportar nada de un posible contenido. Es cierto que en las explicaciones sobre las competencias plurilingües y pluriculturales, en especial el savoir être, se puede intuir el cambio de paradigma, algo de una "Tertiärkultur(alität)" (Földes 513) o de la "dritte Ordnung der Interkulturalität" (Wierlacher b, 261), sobre todo al descubrir neologismos como el de Heimatkultur en el siguiente contexto:

"se puede afirmar que el conocimiento de una lengua y de una cultura extranjeras no siempre supone ir más allá de lo que pueda ser etnocéntrico con relación a la lengua y a la cultura "nativas», sino que incluso puede tener el efecto contrario (no es raro que el aprendizaje de una lengua y el contacto con una cultura extranjera refuerce los estereotipos e ideas preconcebidas, y no los reduzca)." (Consejo de Europa 2002, 132)

Por el contrario, elLibro Blanco sobre el Diálogo intercultural sí dedica un pequeño capítulo sobre enseñanza y aprendizaje de competencias interculturales, pero se enmarca dentro de un conjunto de objetivos ideológicos y políticos cuya clave es la ciudadanía democrática (Consejo de Europa 2008, 34-43). No encontramos ninguna aportación sobre la naturaleza de dicho aprendizaje, en cambio se observa una clara instrumentalización del término.

Otros documentos como la Estrategia de Lisboa (Parlamento Europeo) - que incluye las lenguas extranjeras entre las nuevas habilidades que se pretenden reforzar - y Europa 2020(Comisión Europea) persiguen la misma línea político-económica y democrática para fortalecer la posición de Europa en el mundo.

El Comunicado de Lovaina (Conferencia de Ministros Europeos) reafirma las líneas de Bolonia, pero no trata el tema de las lenguas y culturas, y tampoco la competencia intercultural.

En lo que concierne al ámbito alemán, destacamos la Gesetz zur Regelung von Partizipation und Integration en el Estado de Berlín, puesto que se trata de la primera ley Federal en la que se regula la participación y la integración de las personas de origen inmigrante:

"Artikel 1

4 Gleichberechtigte Teilhabe und interkulturelle Öffnung

(3) Interkulturelle Kompetenz ist eine auf Kenntnissen über kulturell geprägte Regeln, Normen, Wertehaltungen und Symbole beruhende Form der fachlichen und sozialen Kompetenz. Der Erwerb von und die Weiterbildung in interkultureller Kompetenz sind für alle Beschäftigten durch Fortbildungsangebote und Qualifizierungsmaßnahmen sicherzustellen. Die interkulturelle 
Kompetenz soll bei der Beurteilung der Eignung, Befähigung und fachlichen Leistung im Rahmen von Einstellungen und Aufstiegen der Beschäftigten im öffentlichen Dienst grundsätzlich berücksichtigt werden."

La competencia intercultural se percibe aquí como un elemento - cognitivo - más en la cualificación de los empleados en los servicios públicos. Si bien es cierto que esta definición responde a una necesidad social real - porque se es consciente de ella - , se "sabe" algo sobre otras culturas, no se interactúa con ellas.

Sin embargo, una nueva ley promulgada el 14 de febrero de 2012 por el Land Nordrhein-Westfalen define la competencia intercultural en su artículo 4 (2) con vistas a una interacción:

„Interkulturelle Kompetenz im Sinne dieses Gesetzes umfasst

1. die Fähigkeit, insbesondere in beruflichen Situationen mit Menschen mit und ohne Migrationshintergrund erfolgreich und zur gegenseitigen Zufriedenheit agieren zu können“.

En consonancia con las nuevas líneas en materia de competencia intercultural en la Unión Europea, también en España se abre un camino hacia la integración de los nuevos planteamientos. A continuación revisamos las leyes educativas vigentes en España desde los años 70.

\subsection{Normativa española: hacia el reconocimiento de la interculturalidad}

La interculturalidad en España aparece en los años noventa en la universidad, fundamentalmente en Ciencias de la Educación y Ciencias del Lenguaje como consecuencia del aumento de la población inmigrante. La escolarización de los niños procedentes de otras culturas ha supuesto un aumento de la diversidad en el aula, al contrario que en el resto de Europa donde el flujo de emigrantes se produce durante varias décadas. Este cambio tan rápido conllevó la dificultad de aceptar al diferente, al extranjero con su propia identidad. En consecuencia, surge el temor a perder la propia identidad por lo que se defiende la propia cultura, fomentándose una visión etnocentrista.

En el ámbito de la educación, el gobierno español recoge en diversas leyes su preocupación por la nueva tipología de alumnos procedentes de otras culturas. De ellas nos referiremos únicamente a aquellas que hacen referencia o que han introducido cambios con respecto al tema de nuestra investigación.

En 1970 la Ley General de Educación y Financiamiento de la Reforma Educativa (LGE), como esboza el propio nombre, no hace referencia 
explícita a la interculturalidad ni aparece el término cultura. Esta reforma intentó modernizar la estructura del sistema educativo para adecuarlo a una sociedad en proceso de cambios. Se centra especialmente en la financiación de los equipamientos, garantiza el derecho a la educación de los extranjeros, en las mismas condiciones que los españoles. En el Artículo cuarenta y ocho se prevén cursos para los extranjeros que permitan a éstos, entre otras cosas, "informarse de la cultura española".

En el TITULO PRELIMINAR, Artículo primero, encontramos la única referencia al espíritu internacional: "Tres. La incorporación de las peculiaridades regionales, que enriquecen la unidad y el patrimonio cultural de España, así como el fomento del espíritu de comprensión y de cooperación internacional."

Quince años después aparece la LODE (Ley Orgánica, Reguladora del Derecho a la Educación, 1985), que deroga la LOECE (1980, Ley Orgánica reguladora del Estatuto de Centros Escolares, no aporta novedades al tema de investigación).Crea nuevos organismos como el consejo escolar y articula medidas compensatorias para los alumnos que las necesiten por razones socioculturales.

La tercera reforma importante se produce en 1990 con la llegada de la LOGSE (Ley Orgánica General del Sistema Educativo), primera ley de educación en la democracia. Pretendía ser una ley con grandes reformas, ajustadas al tiempo social y político caracterizado por un flujo importante de población inmigrante, pero las reformas planteadas no fueron suficientes. Respecto al lenguaje legal introduce algunos cambios: no se refiere al hecho de ser extranjero sino a aspectos culturales. Por primera vez se introducen conceptos como diferencias culturales, de carácter étnico, de origen geográfico, aunque no aparece el vocablo intercultural. El artículo 1 se expresa en los siguientes términos: "g) La formación para la paz, la cooperación y la solidaridad entre los pueblos". Sin embargo, esta ley emprende una ambiciosa reforma pero, como manifiesta Puelles Benítez, "no fue acompañada de una política acertada de formación del profesorado, ni de la formación inicial ni de la formación continua" (7).

En 1995 la LOPEG (Ley Orgánica de la Participación, Evaluación y Gobierno), reforma algunos puntos de la LOGSE: se reafirma el derecho a la educación sin discriminación y consolida la autonomía de los centros educativos. Al año siguiente se aprueba el Real Decreto 299/1996, de 28 de febrero (BOE de 12-3-96), en el que se hace referencia a la compensación educativa, a la erradicación del analfabetismo y a la adquisición de las lenguas de acogida. En esta ley se recogen dos principios fundamentales en la educación intercultural. Por una parte se ofrece apoyo a los sectores de población más desfavorecidos y se alude a la necesidad de buscar solución ante situaciones de desigualdad, sin 
necesidad de perder la identidad y cultura, por otro lado, se suscitan actitudes de respeto y tolerancia hacia otras culturas minoritarias.

En 2002 aparece la LOCE (Ley Orgánica de la Calidad de la Educación), que se centra especialmente en los aspectos académicos. Se equipara el acceso a la educación de los extranjeros a la de los alumnos españoles y se refiere a medidas compensatorias, en caso de necesidad, para los alumnos inmigrantes. Es importante conocer "los aspectos básicos de la cultura y la historia y respetar el patrimonial artístico y cultural; conocer la diversidad de culturas y sociedades, a fin de poder valorarlas críticamente y desarrollar actitudes"(10).

La última ley, vigente en la actualidad, se promulga en el año 2006, la LOE, una ley que funde la LOGSE, la LOPEG y la LOCE, aunque mantiene la estructura de la LOGSE. De acuerdo con las exigencias del Espacio Europeo de Enseñanza Superior, esta ley acomete una nueva reforma en la formación del profesorado. Los objetivos son ofrecer una educación de calidad y para ello es necesaria la implicación de toda la comunidad educativa. En el artículo $1^{\circ}$ se expone que el sistema educativo español se inspira en los siguientes principios:

"a) La calidad de la educación para todo el alumnado, independientemente de sus condiciones y circunstancias.

b) La equidad, que garantice la igualdad de oportunidades, la inclusión educativa y la no discriminación y actúe como elemento compensador de las desigualdades personales, culturales, económicas y sociales, con especial atención a las que deriven de discapacidad."

El objetivo de esta ley es atender a la diversidad educativa, independientemente del género, se trata de conseguir que todos los ciudadanos tengan las mismas oportunidades y alcancen el máximo desarrollo en sus capacidades, individuales, sociales, intelectuales, culturales y emocionales. En el artículo 2. Fines, se manifiesta:

"1. El sistema educativo español se orientará a la consecución de los siguientes fines: [...]g) La formación en el respeto y reconocimiento de la pluralidad lingüística y cultural de España y de la interculturalidad como un elemento enriquecedor de la sociedad."

Encontramos escasas referencias a la interculturalidad y se centra más en una educación dirigida al respeto y a la diferencia, la atención a la diversidad sin diferenciar el país.

En España la educación está transferida a las Autonomías, que tienen libertad para adaptar lo recogido en la legislación estatal. Nos referimos brevemente a las novedades en la legislación de la Comunidad de Andalucía donde se crea el Plan Integral para la Inmigración. El primero para el periodo 2001-2004 y el segundo para el periodo 2006-2009. En 
relación a la educación intercultural, el I Plan Integral para la Inmigraciónen Andalucía(Junta de Andalucía) se pronuncia en estos términos:

"La interculturalidad supone [...] una educación que se destina a la generalidad de la población y no sólo a las minorías nacionales o inmigrantes [...] supone enseñar a la ciudadanía a mirar a la otra persona con una óptica distinta para comprender cómo piensa y cómo siente [...] implica también facilitar la igualdad de oportunidades para los miembros de las minorías culturales desfavorecidas, adquiriendo un carácter compensador." (86)

La interculturalidad se presenta aquí como un punto de partida, sin embargo sería importante dar respuesta a la siguiente pregunta clave: ¿Cómo se forma a los futuros profesores para que adquieran competencias interculturales que ayuden a construir esa realidad intercultural?

\subsection{La competencia intercultural en el Espacio Europeo de Enseñanza Superior en el Libro Blanco del Título de Grado de Lenguas Modernas y sus Literaturas}

Desde el Espacio Europeo de Educación Superior se han impulsado reformas en las universidades españolas, se proponen nuevos paradigmas formativos para los futuros egresados y docentes y se reorientan las políticas educativas a fin de llevar a cabo el proceso de Bolonia (EURYDICE). El Ministerio de Educación y Ciencias, las Universidades españolas y la Agencia Nacional de Evaluación de la calidad y Acreditación (ANECA) han consensuado un modelo de los aspectos más relevantes para el diseño de los nuevos Títulos de Grado, recogidos en los Libros Blancos de las titulaciones.

En relación a la formación en competencia intercultural de los docentes, para el Grado de Lenguas Modernas y sus Literaturas, descrito en el Libro Blanco de Lengua, Literatura y Civilización, en el apartado 2 referido a la justificación de la denominación del grado, se describe lo siguiente:

"El Grado en Lenguas Modernas y sus Literaturas agrupa en una misma titulación distintas lenguas extranjeras que constituyen un ámbito de estudio con entidad propia tradicional, tanto en la universidad española, como en las europeas y que posee unos perfiles científicos bien definidos y unos fines laborales variados, aunque convergentes: aparte de la enseñanza, permite la apertura cultural en distintos sectores de intermediación lingüística y cultural, como profesionales de institutos de cultura, bibliotecas, centros de investigación; actividad en el campo editorial y en el de la traducción; relaciones internacionales, representación diplomática, administraciones públicas, gestión cultural, turismo, etc." (ANECA 433). 
Sin embargo, en el Libro Blanco no hemos encontrado referencias directas a las competencias interculturales, es más, brillan por su ausencia.El cambio de paradigma pensado y anunciado en los documentos que determinan el Espacio Europeo de Enseñanza Superior no se ha efectuado aún.

\section{Conclusiones}

Nuestro estudio revela que hay una discrepancia entre la investigación y la aplicación práctica de los resultados. Esta discrepancia se refleja en los documentos europeos y españoles, pues a pesar de que existen reflexiones sobre la diversidad cultural, expresada como interculturalidad o educación intercultural, ésta se presenta como una sugerencia a adoptar en el aula, no como una obligación. Ello conlleva que el diseño y la puesta en práctica de los nuevos modelos, en proceso de desarrollo, sean responsabilidad del colectivo docente.

La interculturalidad se describe como una actitud y forma de pensar la enseñanza y no como un contenido preciso y definido. Creemos que el vaivén legislativo existente hasta este momento no ha sido suficiente para lograr una estabilidad legislativa. En los últimos 25 años se han creado en España diversas leyes educativas, pero ninguna de ellas, ni incluso los actuales Libros Blancos, dan pautas reales para desarrollar en la formación de los estudiantes la interculturalidad, más aún, el éxito de su aplicación está en función de la implicación del centro educativo y del profesorado.

En un reciente estudio (Ruiz, Heinsch y Ruiz 266) sobre los nuevos perfiles del docente de lenguas extranjeras (especialmente de alemán), se concluye que ni en España ni en Alemania los programas de estudio analizados, conducentes a un título de máster en Español y Alemán (respectivamente) como Lengua Extranjera, integran plenamente los instrumentos y medios propuestos y recomendados por la Unión Europea; asimismo se llama la atención sobre la orientación monocultural de la formación frente a la realidad social claramente multicultural.

En consecuencia, ante la nueva realidad socioeducativa y profesional, estrechamente vinculada al concepto de empleabilidad, que demanda un profesorado formado en competencias interculturales, capaz de proporcionar una enseñanza de calidad y hacer frente a situaciones complejas que se generan en el proceso de enseñanza-aprendizaje, abogamos por una sólida formación docente sobre el tema que nos ocupa, más allá de los contenidos tradicionales de la filología alemana, como ya lo insinuaba Krumm (414) hace diez años, y por el tratamiento de la cultura como un elemento generador de riqueza que participa e incide en el desarrollo de las personas. 
La interculturalidad no sólo tiene que ver con la mirada hacia el otro país y cultura, sino también hacia lo propio, pues dentro de un mismo grupo cultural existe la experiencia de lo ajeno.

\section{Referencias bibliográficas:}

ANECA, Libro Blanco.Título de Grado en Estudios en el ámbito de la Lengua, Literatura, Cultura y Civilización,Madrid, ANECA, 2005.

ASUNCIÓN-LANDE,Nobleza,"Estudios Interculturales y educación. Bases Teóricas", 1997 URL:http://www.uv.mx/dei/P publicaciones/BasesTeoricas/.(411-2012).

BARRO, Ana; JORDAN, Shirley; RoBERTS, Celia, "La práctica cultural en la vida cotidiana: el estudiante de idiomas como etnógrafo", en BYRAM M. y FLEMING, M., Perspectivas interculturales en el aprendizaje de idiomas, Madrid, Cambridge University Press, 2001, págs. 82-103.

BATES, David Gregory; PLOG, Fred, Cultural anthropology, New York, McGraw-Hill, 1990.

CASANOVA, María Antonia, "Interculturalidad y educación. Reflexiones para la actuación en los centros docentes", El profesorado y el cambio educativo. Jornadas Educativas 15- 16 de marzo, Madrid, Centro de Innovación Educativa 2002, págs. 9-32.

COMISIÓN EUROPEA, "EUROPA 2020 Una estrategia para un crecimiento inteligente, sostenible e integrador", 3.3.2010. URL: http://eurlex.europa.eu/LexUriServ/LexUriServ.do?uri=COM:2010:2020:FI $\mathrm{N}: \mathrm{ES}: \mathrm{HTML} \cdot(\mathbf{1 1 - 2 - 2 0 1 3 ) .}$

CONFERENCIA DE MINISTROS EUROPEOS RESPONSABLES DE EDUCACIÓN SUPERIOR, Comunicado de Lovaina, "El proceso de Bolonia 2020 - El Espacio Europeo de Educación Superior en la nueva década. Comunicado de la Conferencia de Ministros europeos responsables de educación superior, Lovaina / Louvain-la-Neuve, 28-29 de abril de 2009", 2009. URL: http://www.crue.org/export/sites/Crue/procbolonia/documentos/ant ecedentes/Comunicado Lovaina Ministerio es.pdf . (11-2-2013).

CONSEJO DE EUROPA, Libro Blanco sobre el Diálogo Intercultural, EstrasburgoMadrid, $2008 . \quad$ URL: http://www.coe.int/t/dg4/intercultural/Source/Pub White Paper/W hitePaper ID SpanishVersion.pdf (4-12-2012).

CONSEJO DE EUROPA, Marco común europeo de referencia para las lenguas: enseñanza. Aprendizaje y evaluación, Madrid, Ministerio de Educación, Cultura y Deporte, 2002. http://cvc.cervantes.es/obref/marco/cvc mer.pdf.

DIE LANDESREGIERUNG NORDRHIEN-WESTFALEN, Gesetz zur Förderung der gesellschaftlichen Teilhabe und Integration inNordrhein-Westfalen und zur Anpassung anderer gesetzlicher Vorschriften.URL: https://recht.nrw.de/lmi/owa/br vbl detail text?anw nr=6\&vd id= 13197\&ver=8\&val=13197\&sg=0\&menu=1\&vd back=N (3-2-2013). 
ENGEL, Ulrich et al. (ed.), Mannheimer Gutachten zu ausgewählten Lehrwerken Deutsch als Fremdsprache, 1, Heidelberg,Julius Groos, 1977.

EURYDICE, La integración escolar del alumnado inmigrante en Europa, BruselasUnidad Europea de Eurydice,Eurydice 2004.

FöLDES, Csaba,"Black Box ,Interkulturalität'. Die unbekannte Bekannte (nicht nur) für Deutsch als Fremd-/Zweitsprache. Rückblick, Kontexte und Ausblick", en Wirkendes Wort59 (2009) 3, págs. 503-525.

GESETZ ZUR REGELUNG VON PARTIZIPATION UND INTEGRATION (PARTINTG), en Gesetz- und Verordnungsblatt für Berlin, Berlin, 28. Dezember 2010. URL: http://www.berlin.de/imperia/md/content/lb-integration-

migra-

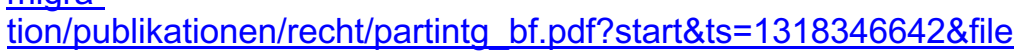
$=$ partintg bf.pdf (2-12-2012).

GöHRING, Heinz,"Kontrastive Kulturanalyse und Deutsch als Fremdsprache“, en Jahrbuch Deutsch als Fremdsprache 1(1975), págs. 80-92.

HOFSTEDE, Geert,Cultures and Organizations: Software of the Mind,Nueva York, Mc Graw-Hill, 1991.

IGLESIAS CASAL, Isabel,"Construyendo la competencia intercultural: sobre creencias, conocimientos y destrezas", en Carabela 54 (1999), págs. 5-28.

JUNTA DE ANDALUCÍA, I Plan Integral para la inmigración en Andalucía,Sevilla, Consejería de Gobernación. D.G. de Coordinación de Políticas Migratorias 2001-2004.

KROEBER, AlfredLouis; KLUCKHOHN, Clyde,Culture: A critical review of concepts and definition,New York, Vintage Books, 1952.

KRUMM, Hans-Jürgen, „Interkulturelle Fremdsprachendidaktik“, en WIERLACHER, Alois; BOGNER, Andrea (ed.), Handbuch interkulturelle Germanistik, Stuttgart Weimar,J. B. Metzler, 2003, págs. 413-417.

KUMPER, Adam,Cultura. La versión de los antropólogos, Barcelona, Paidós, 2001.

LEY ORGÁNICA de Ordenación General del Sistema Educativo (LOGSE), BOE nº 1238, de 4 octubre de 1990.

LEY ORGÁNICA de Calidad de la Educación, BOE n 307 de 24 de diciembre de 2002.

LEY ORGÁNICA General de Educación y Financiamiento de la Reforma Educativa, BOE no 187, de 6 de agosto de 1970.

LEY ORGÁNICAreguladora del Estatuto de Centros Escolares, BOE n 154, de 27 de junio de 1980.

LEY ORGÁNICAreguladora del Derecho a la Educación (LODE), BOE n 159, de 4 de julio de 1985.

LEY ORGÁNICA de Participación, Evaluación y Gobierno de los Centros Docentes, BOE n 278 de 21 de noviembre de 1995.

LLUCH BLAGUER, Xavier; SALINAS CATALÁ,Jesús, La diversidad cultural en la práctica educativa. Materiales para la formación del profesorado en Educación Intercultural,Madrid, MEC, 1996.

MALINOWSKI, Bronislaw,Una teoría científica de la cultura, Barcelona, Edhasa 1970, [1944].

MANZINI, V.,Multiculturalidad, Interculturalidad: Conceptos y estrategias, Bolonia: Universidad de Bolonia, 2001.

PARLAMENTO EUROPEO, Estrategia de Lisboa, "Consejo Europeode Lisboa23 Y 24 de Marzo 2000. Conclusiones de la Presidencia", 2000. 
URL: http://www.europarl.europa.eu/summits/lis1 es.htm . (11-22013).

Puelles BeníteZ, Manuel, "Las grandes leyes educativas de los últimos doscientos años", en Participación Educativa, 7 (2008), págs.9-17.

RATHJE, Stefanie, „Interkulturelle Kompetenz - Zustand und Zukunft eines umstrittenen Konzepts", en Zeitschrift für interkulturellen Fremdsprachenunterricht 11, 3 (2006), 21 págs. URL: http://zif.spz.tu-darmstadt.de/jg-113/docs/Rathje.pdf (4-12-2012).

RODRIGO ALSINA, Miguel, "Elementos para una comunicación intercultural", en Espacios de la interculturalidad, 1997.URL:http://www.cidob.es/Castellano/Publicaciones/Afers/rodrig o.htm,. (4-10-2012).

RUIz SÁNCHEZ, Ana; HEINSCH, Bárbara;RUIZ SÁNCHEZ, Aránzazu, "Neue Profile für DaF an der Schwelle zu einer neuen Hochschullandschaft", en Estudios Filológicos Alemanes (2012) 24, págs. 255-267.

SPITZER, Leo,"Das Eigene und das Fremde. Über Philologie und Nationalismus“, en Die Wandlung 1, (1945/46), págs. 576-594.

RUIZ ROMÁN, Cristóbal, Educación intercultural: Una visión crítica de la cultura, Barcelona, Octaedro 2003.

THOMAS, Alexander,"Lernen und interkulturelles Lernen“, enWIERLACHER, Alois; BOGNER, Andrea (ed.), Handbuch interkulturelle Germanistik, Stuttgart Weimar, J. B. Metzler, 2003, págs. 276-287.

TYLOR, Edward Burnett, "La Ciencia de la Cultura", en KAHN, J. S., El Concepto de Cultura: Textos Fundamentales, Barcelona, Anagrama 1975, [1871].

UNIÓN EURPEA, Europa 2020. URL: http://europa.eu/ legislation summaries/ employment and social policy/eu2020/em0028 es.htm (5-12-2012).

WIERLACHER, Alois (a), "Interkulturelle Germanistik. Zu ihrer Geschichte und Theorie. Mit einer Forschungsbibliographie", en WIERLACHER, Alois / BOGNER, Andrea (ed.), Handbuch interkulturelle Germanistik, Stuttgart Weimar, J. B. Metzler, 2003, págs. 1-45.

WIERLACHER, Alois, (b), "Interkulturalität", en WieRLACHER, Alois / BOgneR, Andrea (ed.), Handbuch interkulturelle Germanistik. Stuttgart Weimar, J. B. Metzler, 2003, págs. 257-264. 